\title{
Prognostic factors for aorta remodeling after thoracic endovascular aortic repair of complicated chronic DeBakey IIIb aneurysms
}

\author{
Suk-Won Song, MD, PhD, ${ }^{\text {a }}$ Tae Hoon Kim, MD, ${ }^{a}$ Sun-Hee Lim, RN, ${ }^{a}$ Kwang-Hun Lee, MD, PhD, \\ Kyung-Jong Yoo, MD, $\mathrm{PhD},{ }^{c}$ and Bum-Koo Cho, $\mathrm{MD}, \mathrm{PhD}^{\mathrm{d}}$
}

Objectives: The use of thoracic endovascular aortic repair (TEVAR) for chronic DeBakey III type b (CDIIIb) aneurysms is controversial. We analyzed the potential prognostic factors affecting aorta remodeling after this procedure.

\begin{abstract}
Methods: A total of 20 patients with CDIIIb aneurysms underwent TEVAR, with full coverage of reentry tears at the descending thoracic aorta. The potential factors affecting false lumen (FL) remodeling were analyzed, including reentry tears (communicating channels visible on the computed tomography angiogram), large intimal tears below the stent graft ( $\geq 2$ consecutive axial cuts on the computed tomography angiogram), visceral branches arising from the FL, and intercostal arteries (ICAs) arising from the FL.
\end{abstract}

Results: All the patients had uneventful in-hospital courses; 2 patients $(10 \%)$ required reintervention during the follow-up period. Thirteen patients $(65 \%)$ had complete thrombosis of the FL at stent graft segment. Compared with the complete thrombosis group, the partial thrombosis group had more reentry tears $(1.8$ vs $2.3, P=.48)$, large intimal tears $(0.8$ vs $1.7, P<.05)$, visceral branches arising from the FL $(1.2$ vs $2.3, P<.05)$, and ICAs arising from the FL (3.8 vs 5.1, $P=.35$ ). Reentry tears, visceral branches, and ICAs from the FL were significant negative prognostic factors for FL shrinkage $(P<.05)$.

Conclusions: Although reentry tears above the celiac trunk were fully covered, the visceral branches and ICAs from the FL and all communicating channels below the celiac trunk kept the FL pressurized and were unfavorable prognostic factors for aorta remodeling after TEVAR for CDIIIb aneurysms. (J Thorac Cardiovasc Surg 2014;148:925-33)

Supplemental material is available online.

The ideal treatment of patients with chronic DeBakey III (CDIII) aortic dissections remains controversial. The optimal medical therapy of anti-impulse treatment has been used in most patients, and selective open or endovascular repair has been used for complicated chronic cases,

\footnotetext{
From the Departments of Thoracic and Cardiovascular Surgery ${ }^{\mathrm{a}}$ and Interventional Radiology, ${ }^{\mathrm{b}}$ Gangnam Severance Hospital, Yonsei University College of Medicine, Seoul, Republic of Korea; Department of Cardiovascular Surgery, ${ }^{\mathrm{c}}$ Yonsei Cardiovascular Hospital, Severance Hospital, Yonsei University College of Medicine, Seoul, Republic of Korea; and The Korea Heart Foundation, ${ }^{\mathrm{d}}$ Seoul, Republic of Korea.

Disclosures: Authors have nothing to disclose with regard to commercial support.

Drs Song and Kim contributed equally to this work.

Read at the 94th Annual Meeting of The American Association for Thoracic Surgery, Toronto, Ontario, Canada, April 26-30, 2014.

Received for publication April 7, 2014; revisions received May 8, 2014; accepted for publication May 16, 2014; available ahead of print June 18, 2014.

Address for reprints: Kwang-Hun Lee, MD, PhD, Department of Interventional Radiology, Gangnam Severance Hospital, Yonsei University College of Medicine, 612 Eonjuro, Gangnam-gu, Seoul 135-720, Republic of Korea (E-mail: doctorlkh@ yuhs.ac).

$0022-5223 / \$ 36.00$

Copyright (C) 2014 by The American Association for Thoracic Surgery

http://dx.doi.org/10.1016/j.jtcvs.2014.05.040
}

such as those involving aneurysmal degeneration, malperfusion, persistent pain, or rupture. Although recent series have suggested thoracic endovascular aortic repair (TEVAR) as a promising treatment of acute, complicated type B aortic dissections, ${ }^{1,2}$ outcome studies of TEVAR-treated CDIII aneurysms have been primarily retrospective, single-center experiences involving heterogeneous cohorts with various operative indications and different strategies.

Open surgery for CDIII aneurysms has been associated with substantial morbidity and mortality. ${ }^{3,4}$ In light of the success of TEVAR in patients with degenerative aneurysms, this therapy offers an attractive alternative to open surgery for CDIII aneurysms. ${ }^{5,6}$ However, several issues also mitigate against the use of TEVAR in these patients. The presence of a mature, rigid dissection flap, and multiple reentry tears have been thought to potentiate failure of endovascular therapy, and the long-term durability of endovascular management remains uncertain. ${ }^{7}$

The primary aim of managing CDIII aneurysms is to avoid death from aortic rupture. This clinical endpoint has frequently been associated with the ability of TEVAR to trigger favorable aortic remodeling, an important outcome predictor of false lumen (FL) patency. ${ }^{8,9}$

Although the results of TEVAR for CDIII type a (CDIIIa) aneurysms have been exciting, concerns regarding the use 


\section{Abbreviations and Acronyms \\ CDIII = chronic DeBakey III \\ CDIIIa = chronic DeBakey III type a \\ $\mathrm{CDIIIb}=$ chronic DeBakey III type $\mathrm{b}$ \\ CTA = computed tomography angiogram \\ FL $\quad$ false lumen \\ ICA $=$ intercostal artery \\ SCI = spinal cord ischemia \\ TEVAR $=$ thoracic endovascular aortic repair}

of this technique in patients with CDIII type b (CDIIIb) aneurysms remain, including concerns about the precipitation of retrograde dissection or visceral ischemia. ${ }^{10,11}$

The present study investigated the prognostic factors for aorta remodeling after TEVAR for complicated CDIIIb aneurysms.

\section{METHODS \\ Patient Population}

The institutional review board at the Gangnam Severance Hospital, Yonsei University College of Medicine (Yonsei institutional review board no. 3-2012-0259) approved the present study. A prospectively maintained endovascular aortic registry was queried for all patients undergoing TEVAR from May 2012 to April 2013.

Patients treated for degenerative and trauma-related pathologic entities (including aneurysms, penetrating ulcers, atheromatous disease, pseudoaneurysms, and traumatic transections) were excluded. Patients with dissection-related indications were reviewed further, and only those undergoing elective procedures for chronic ( $>3$ months after the diagnosis of acute aortic dissection) aneurysmal degeneration of descending aortic dissections extending to the abdominal aorta (CDIIIb) were included. The patients with dissecting aneurysms confined to the descending thoracic aorta (CDIIIa) were excluded. Although acute proximal dissections were not included, patients previously treated for type A dissections who had residual CDIIIb aneurysms were included. The indications for TEVAR included newly developed, continuing back pain, and aneurysmal degeneration (maximal thoracic aneurysm diameter $\geq 5.5 \mathrm{~cm}$ or a documented growth rate of 0.5 $\mathrm{cm}$ within 6 months seen on serial computed tomography angiograms [CTA]). The demographics, comorbidities, procedure-specific details, reinterventions, and complications were collected from the medical records.

\section{Clinical Practice}

A standardized treatment algorithm was used throughout the study period for patient selection and procedural conduct. All operations were performed electively with the patient under general anesthesia in a hybrid operating room. The need for perioperative adjuncts (eg, spinal drainage, carotid-subclavian bypass, open or percutaneous vessel access) were at the discretion of the attending surgeon and interventional radiologist.

All patients received systemic heparin $(100 \mathrm{U} / \mathrm{kg})$ to achieve an activated clotting time of more than 300 seconds. Devices were oversized $10 \%$ to $15 \%$, relative to the diameter of the undissected aorta proximal to the dissection, using tapered stent grafts. At the beginning of study period, the distal landing zone was in the mid-descending thoracic aorta (group 3). However, for the remaining cases, the coverage length was selected to exclude the entire dissected thoracic aorta and all fenestrations (group 2). Our current practice has been for more aggressive aortic coverage (ie, we generally treat down to the celiac artery in patients with juxtaceliac fenestrations (group 1; Figure 1). In some cases, we have covered the celiac trunk, when backflow from the superior mesenteric artery was expected. Technical success was defined as endograft deployment at the intended aortic segment, with the absence of antegrade flow into the FL. Compliant balloon angioplasty of the proximal stent graft was selectively performed only in the event of type Ia endoleaks. Spinal cord ischemia (SCI) was defined as any new lower extremity motor and/or sensory deficit not attributable to intracranial pathologic features, peripheral neuropathy, or neuropraxia. The diagnosis was determined by the treating physician and confirmatory imaging.

\section{Degree of FL Thrombosis}

The degree of FL thrombosis was analyzed using precontrast, arterial, and delayed phases of postoperative CTA (3-mm-thick axial slices). It was classified as partial thrombosis if both flow and thrombus were present and as complete thrombosis if no flow was present at the level of the stent graft. ${ }^{12}$

\section{Remodeling Analysis}

Aorta remodeling after TEVAR was analyzed, as shown in Figure E1. The preoperative FL diameter was marked as "a," and the postoperative FL diameter was marked as "b." The measurements of "a" and "b" were performed at the level of the maximal descending thoracic aortic aneurysm. We sought to find risk factors affecting the FL ratio (b/a). If $>2$ CT scans had been performed after hospital discharge, the most recent image was used to determine the ratio. If an aortic segment had been subjected to repeated aortic procedures, the patient was excluded from additional FL diameter assessments. Postoperative surveillance was determined using a protocol of imaging before discharge, at 3 and 6 months after discharge, and annually thereafter. We also analyzed the relationship of the degree of FL thrombosis with the FL ratio.

\section{Anatomic Factors}

The maximum diameters of the thoracic aorta, thoracic FL, abdominal aorta, and abdominal FLs were measured on the preoperative CTAs. The following parameters were measured during postoperative CTAs before patient discharge by 2 experienced vascular radiologists: residual reentry tears were counted below the stent graft; large intimal tears were counted, as seen in $\geq 2$ CTA axial cuts below the stent graft; the numbers of intercostal arteries (ICAs) and visceral arteries from the FL were counted; and the maximum diameters of the FLs were determined at the last follow-up CTAs.

\section{Statistical Analysis}

The categorical variables were summarized using frequencies and percentages. Continuous variables were analyzed using the mean and standard deviations, if they were normally distributed. Comparisons of patient- or procedure-related characteristics in subgroup analyses were performed using Fisher's exact tests or independent $t$ tests. Variables with $P<.1$ on univariate analysis were included in the multivariate model, which was refined using stepwise backward binary logistic regression. The relationship between the FL ratios and patient variables was analyzed using Spearman's rank correlation and linear regression analysis. All statistical analyses were performed using the Statistical Package for Social Sciences, version 20, software (SPSS, Chicago, Ill).

\section{RESULTS}

The Gangnam Severance TEVAR registry $(\mathrm{n}=103)$ identified 20 patients $(19 \%)$, who had undergone elective TEVAR for the treatment of CDIIIb aneurysms from May 2012 to April 2013. The mean interval from the diagnosis of the acute dissection to TEVAR was $18.1 \pm 11.9$ months (range, 4-47). Most patients were men $(n=17$ [85\%]; mean 

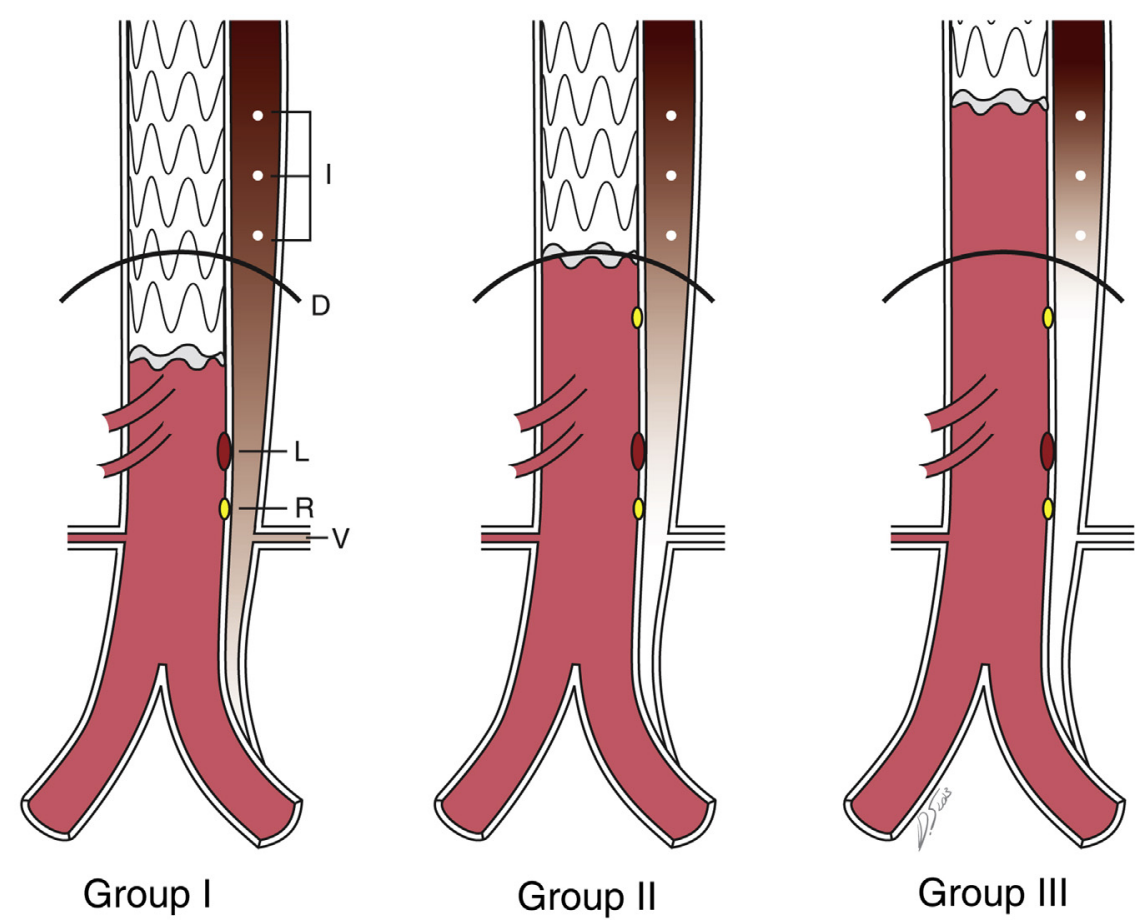

FIGURE 1. Schematic diagram of anatomic factors affecting false lumens after thoracic endovascular aortic repair. Groups were classified according to the distal landing zone: group I, covered to the celiac trunk; group II, whole descending thoracic aorta; group III, mid-descending thoracic aorta. I, Intercostal artery from the false lumen; $D$, diaphragm; $L$, large intimal tear; $R$, reentry tear; $V$, visceral branch from the false lumen.

age, 50.2 years), and the mean follow-up duration was 10.3 \pm 4.9 months (range, $5-15)$. Ten patients $(50 \%)$ who had undergone surgery for acute type A dissections had large tears at the proximal descending thoracic aorta because they had had retrograde type A dissections or multiple tears.

\section{Procedural Details and Perioperative Outcomes}

A total of 30 stent grafts were deployed in 20 patients, including the Zenith TX2 Proform (Cook, Bloomington, Ind) in 17 patients and the Valiant Captivia (Medtronic, Minneapolis, Minn) in 3 patients. Eleven patients (55\%) needed 1 stent graft, $8(40 \%)$ needed 2 , and $1(5 \%)$ needed 3.

Primary technical success, defined as the successful closure of the primary tear without a type Ia endoleak, was achieved in all cases. None of the patients required conversion to open surgery. Preoperatively placed spinal drains were used in 13 patients $(65 \%)$.

In 11 patients $(55 \%)$, coverage occurred at Ishimaru zones 1 or $2,{ }^{13}$ and all these patients underwent subclavian revascularization with or without a carotid-carotid bypass. The distal landing zone was group 1 or 2 in almost all patients $(\mathrm{n}=18,90 \%$; Figure 1$)$. All patients were treated with covered stent grafts. The mean hospital and intensive care unit stay was 7.5 days and 28.7 hours, respectively, without any in-hospital deaths or neurologic dysfunction. Additional details regarding the outcomes and complications are listed in Table 1.

\section{Degree of FL Thrombosis}

Thirteen patients $(65 \%)$ had complete thrombosis of the FL during the first postoperative CTA performed a mean of 28 days after TEVAR. Univariate analysis was used to evaluate the 14 parameters listed in Table 2. Values of $P<.1$ were obtained for age at the interventional procedure, number of large intimal tears, number of visceral branches partially or totally arising from the FL, and maximum diameter of the aorta and the FL on the abdominal aorta; these were used in the multivariate analysis. Multivariate binary logistic regression analysis revealed that the number of large intimal tears (odds ratio, 11.30; 95\% confidence interval, $1.16-110.40 ; P=.037$ ) was an independent risk factor for partial thrombosis. When we focused on the anatomic characteristics of the dissections and compared them with those of the partial thrombosis group, the number of reentry tears, large intimal tears below the stent graft, visceral branches arising from the FL, and ICAs arising from the FL in the complete thrombosis group was 1.8 versus $2.3(P=.48)$, 0.8 versus $1.7(P<.05), 1.2$ versus $2.3(P<.05)$, and 3.8 versus $5.1(P=.35)$, respectively (Table 2$)$.

\section{Remodeling Analysis}

Remodeling analysis was performed with the FL ratio using the preoperative CTA and last CTA (4.7 months after TEVAR). Univariate linear regression analysis revealed that age, number of reentry tears, visceral branches and 
ICAs from the FL, and preoperative thoracic FL diameters were significant negative prognostic factors predicting FL shrinkage $(P<.05)$. Multivariate linear regression analysis revealed that age $(P=.015)$ and the number of visceral branches and ICAs from the FL $(P=.002)$ were independent risk factors for FL shrinkage (Table 3; Figure 2). The FL ratio of the complete thrombosis group was significantly lower than that of the partial thrombosis group. $(0.70 \pm 0.14$ vs $0.82 \pm 0.07, P=.035$; Table 2 ). The difference in the shrinkage of the descending thoracic aorta, abdominal aorta, and abdominal FL was not significant $(P>.05)$.

\section{Reintervention}

Reintervention was defined as any aortic-related endovascular or open surgical procedure that occurred after the index TEVAR at either the intended treatment zone or remote aortic sites. Two patients $(10 \%$; group 3 ; Figure 2), at the beginning of the present study, had experienced stent graft-induced new intimal tears at 5 months and 1 year after TEVAR, respectively, and had undergone additional TEVAR.

\section{DISCUSSION}

The aim of postaortic dissection TEVAR is to cover the primary entry tear, thereby initiating FL thrombosis and aortic remodeling. Although inconclusive, mounting evidence has suggested that aortic remodeling is significantly associated with improved midterm survival and that persistent FL perfusion is associated with disease progression. $^{14-16}$

Favorable aortic remodeling usually begins within days after TEVAR for acute aortic dissection and continues for $\leq 5$ years. ${ }^{17}$ However, patients with CDIII aneurysms have greater variations in the rate of complete FL thrombosis. ${ }^{14}$ Several explanations have been proposed for this variation. First, according to the current definition, "chronic" dissections include those that have been present for $>2$ weeks, creating significant diversity among individuals within the classification. The INvestigation of STEnt Grafts in Aortic Dissection trial, reporting a total FL thrombosis rate of $92.6 \%$ at 1 year, enrolled patients at a median of 3.5 weeks after the diagnosis of acute aortic dissection. ${ }^{2}$ In contrast, the study with the lowest rate of FL thrombosis $(39 \%)$ treated patients at a mean of 100 weeks after diagnosis, ${ }^{18}$ even after 3 years of follow-up. The difference between these 2 studies suggests the classification of aortic dissections as "acute" or "chronic" is insufficient; the "subacute" classification described in the VIRTUE study (2-4 weeks after the acute event) might be more clinically relevant. ${ }^{19}$ In our study, we defined "chronic" as $>3$ months after the diagnosis of acute aortic dissection. ${ }^{20}$ Second, many reports have described data using mixed populations
TABLE 1. Preoperative, operative, and postoperative outcomes

\begin{tabular}{|c|c|}
\hline Variable & Value \\
\hline Preoperative factors & $20(100)$ \\
\hline Male gender & $17(85)$ \\
\hline Age $(y)$ & $50 \pm 11$ \\
\hline Interval between diagnosis and TEVAR (mo) & $18 \pm 12$ \\
\hline \multicolumn{2}{|l|}{ Comorbidities } \\
\hline HTN & $18(90)$ \\
\hline $\mathrm{DM}$ & $1(5)$ \\
\hline Tobacco use & $14(70)$ \\
\hline Obesity & $14(70)$ \\
\hline Marfan syndrome & $1(5)$ \\
\hline CVA & 0 \\
\hline CAOD & $1(5)$ \\
\hline \multicolumn{2}{|l|}{ Previous aortic surgery } \\
\hline Ascending aorta replacement & $3(15)$ \\
\hline Hemiarch replacement & $1(5)$ \\
\hline Total arch replacement & $5(25)$ \\
\hline Descending aorta replacement & $1(5)$ \\
\hline \multicolumn{2}{|l|}{ Operative details } \\
\hline Preoperative aneurysm diameter $(\mathrm{mm})$ & $52 \pm 11$ \\
\hline \multicolumn{2}{|l|}{ Stent graft type } \\
\hline Cook TX2 Proform* & $17(85)$ \\
\hline Medtronic Valiant Captivia $\dagger$ & $3(15)$ \\
\hline \multicolumn{2}{|l|}{ Procedure details } \\
\hline Cerebrospinal drainage & $13(65)$ \\
\hline \multicolumn{2}{|l|}{ Proximal landing zone } \\
\hline 1 & $4(20)$ \\
\hline 2 & $7(35)$ \\
\hline 3 & $5(25)$ \\
\hline 4 & $4(20)$ \\
\hline \multicolumn{2}{|l|}{ Distal landing zone } \\
\hline I & $8(40)$ \\
\hline II & $10(50)$ \\
\hline III & $2(10)$ \\
\hline \multicolumn{2}{|l|}{ Clinical outcomes and complications } \\
\hline $\operatorname{LOS}(\mathrm{d})$ & $7.5 \pm 5.6$ \\
\hline ICU stay (h) & $28.7 \pm 14.7$ \\
\hline Mortality (\%) & 0 \\
\hline \multicolumn{2}{|l|}{ Complications (n) } \\
\hline Spinal cord ischemia & 0 \\
\hline Stroke & 0 \\
\hline Pulmonary & 0 \\
\hline Cardiac & 0 \\
\hline Renal & 0 \\
\hline Gastrointestinal & 0 \\
\hline Access vessel & $1(5)$ \\
\hline Bleeding (bypass vessel) & $1(5)$ \\
\hline \multicolumn{2}{|l|}{ Endoleak } \\
\hline Ia & 0 \\
\hline II & $2(10)$ \\
\hline Reintervention & $2(10)$ \\
\hline
\end{tabular}


TABLE 2. Risk factors for partial thrombosis in the false lumen after TEVAR

\begin{tabular}{|c|c|c|c|c|c|c|}
\hline \multirow[b]{2}{*}{ Variable } & \multirow{2}{*}{$\begin{array}{c}\text { Complete thrombosis } \\
(\mathrm{n}=13, \mathbf{6 5} \%)\end{array}$} & \multirow{2}{*}{$\begin{array}{l}\text { Partial thrombosis } \\
\quad(n=7,35 \%)\end{array}$} & \multirow[b]{2}{*}{$P$ value } & \multicolumn{3}{|c|}{ Multivariate analysis } \\
\hline & & & & OR & $95 \% \mathrm{CI}$ & $P$ value \\
\hline Age (y) & $53.5 \pm 7.8$ & $44.0 \pm 7.8$ & .018 & & & \\
\hline Male gender & 11 & 6 & .730 & & & \\
\hline HTN & 11 & 7 & .411 & & & \\
\hline Smoking & 9 & 5 & .664 & & & \\
\hline Obesity & 10 & 4 & .336 & & & \\
\hline Reentry & $1.8 \pm 1.7$ & $2.3 \pm 1.1$ & .479 & & & \\
\hline Large intimal tear & $0.8 \pm 0.6$ & $1.7 \pm 0.8$ & .007 & 11.30 & $1.16-110.40$ & .037 \\
\hline Visceral branches from FL & $1.2 \pm 0.8$ & $2.3 \pm 1.0$ & .019 & & & \\
\hline ICAs from FL & $3.8 \pm 2.7$ & $5.1 \pm 3.6$ & .346 & & & \\
\hline Visceral + ICA from FL & $5.0 \pm 2.9$ & $7.4 \pm 3.4$ & .107 & & & \\
\hline \multicolumn{7}{|l|}{ PreMax on $\mathrm{Ab}(\mathrm{mm})$} \\
\hline Aorta & $34.0 \pm 4.8$ & $39.4 \pm 6.2$ & .072 & & & \\
\hline FL & $19.1 \pm 9.8$ & $27.1 \pm 4.2$ & .019 & & & \\
\hline \multicolumn{7}{|l|}{ PreMax on D (mm) } \\
\hline Aorta & $47.9 \pm 7.2$ & $51.1 \pm 9.2$ & .406 & & & \\
\hline FL & $32.4 \pm 10.3$ & $36.1 \pm 7.6$ & .416 & & & \\
\hline FL ratio & $0.70 \pm 0.14$ & $0.82 \pm 0.07$ & .035 & & & \\
\hline
\end{tabular}

Data presented as n or mean \pm standard deviation. OR, Odds ratio; $C I$, confidence interval; HTN, hypertension; FL, false lumen; ICA, intercostal artery; PreMax, preoperative maximal diameter; $A b$, abdominal aorta; $D$, descending thoracic aorta.

with varying disease extents. In patients with CDIIIb aneurysms, involving persistent distal FL perfusion, aorta remodeling has been more difficult than in CDIIIa aneurysms. Rodriguez and colleagues ${ }^{8}$ reported complete thrombosis of the thoracic FL in $64 \%$ of CDIIIa aneurysms and $45 \%$ of CDIIIb aneurysms after TEVAR. We only enrolled patients with CDIIIb aneurysms. Third, perioperative practices can change over time or among centers. ${ }^{21,22}$

TABLE 3. Prognostic factors associated with false lumen ratio

\begin{tabular}{|c|c|c|c|c|}
\hline \multirow[b]{2}{*}{ Variable } & \multirow{2}{*}{$\frac{\text { Univariate }}{P \text { value }}$} & \multicolumn{3}{|c|}{ Multivariate } \\
\hline & & $\mathbf{B}^{*}$ & $95 \%$ CI & $P$ value \\
\hline Age (y) & .039 & -0.508 & -0.013 to -0.002 & .015 \\
\hline Male gender & .081 & & & \\
\hline HTN & .729 & & & \\
\hline Smoking & .134 & & & \\
\hline Obesity & .570 & & & \\
\hline Reentry & .037 & & & \\
\hline Large intimal tear & .229 & & & \\
\hline $\begin{array}{l}\text { Visceral branches } \\
\text { from FL }\end{array}$ & .438 & & & \\
\hline ICAs from FL & .008 & & & \\
\hline $\begin{array}{l}\text { Visceral + ICA } \\
\text { from FL }\end{array}$ & .005 & 0.615 & 0.011 to 0.040 & .002 \\
\hline \multicolumn{5}{|l|}{ PreMax of $\mathrm{Ab}(\mathrm{mm})$} \\
\hline Aorta & .880 & & & \\
\hline FL & .434 & & & \\
\hline \multicolumn{5}{|l|}{ PreMax of D (mm) } \\
\hline Aorta & .173 & & & \\
\hline FL & .007 & & & \\
\hline Complete thrombosis & .340 & & & \\
\hline
\end{tabular}

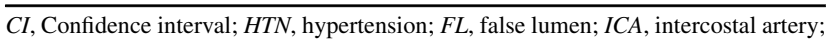
PreMax, preoperative maximal diameter; $A b$, abdominal aorta; $D$, descending thoracic aorta. *Beta co-efficiency (adjusted estimated) for linear regression analysis.
Fourth, a standardized reporting system does not exist, including a lack of consensus regarding definitions of clinical success and concerns about treatment failure. Most reports have focused on FL thrombosis and have relied on the arterial phase of CTA to determine the treatment effect. However, FL can persist in the delayed phase. The absence of contrast in the delayed phase does not indicate thrombosis but denotes a low-flow state. In the present study, we used both the arterial and 3-minute delayed phase; the delayed phase image altered the FL status in 7 cases (35\%) (Figure E2). As evident from these various reports, the reasons for variation in the rates of complete FL thrombosis arise from several sources of cohort heterogeneity. In our study, we aimed for greater patient homogeneity to describe a universal outcome for patients with CDIIIb aneurysms.

The possible mechanisms for persistent FL perfusion include distal reentry tears, retrograde filling flows into the FL, and motion of the dissection flap. ${ }^{11}$ The first mechanism suggests that reentry segments, distal to the stent graft, can provide retrograde perfusion into the thoracic FL and antegrade perfusion into the abdominal FL. Our treatment strategy covers all tears in the descending thoracic aorta to the celiac trunk level to accelerate thrombus formation in the FL. However, this goal is difficult to achieve, particularly in patients with multiple reentry tears located in the abdominal aorta. Multiple tears are commonly seen in CDIIIb aneurysms; $\geq 1$ tear, distal to the orifice of the celiac artery, was present in $62 \%$ of patients in 1 report, ${ }^{23}$ and an average of 2.8 intimal tears was found in another series ${ }^{24}$ allowing continued FL perfusion after TEVAR. In the present study, the primary entry 

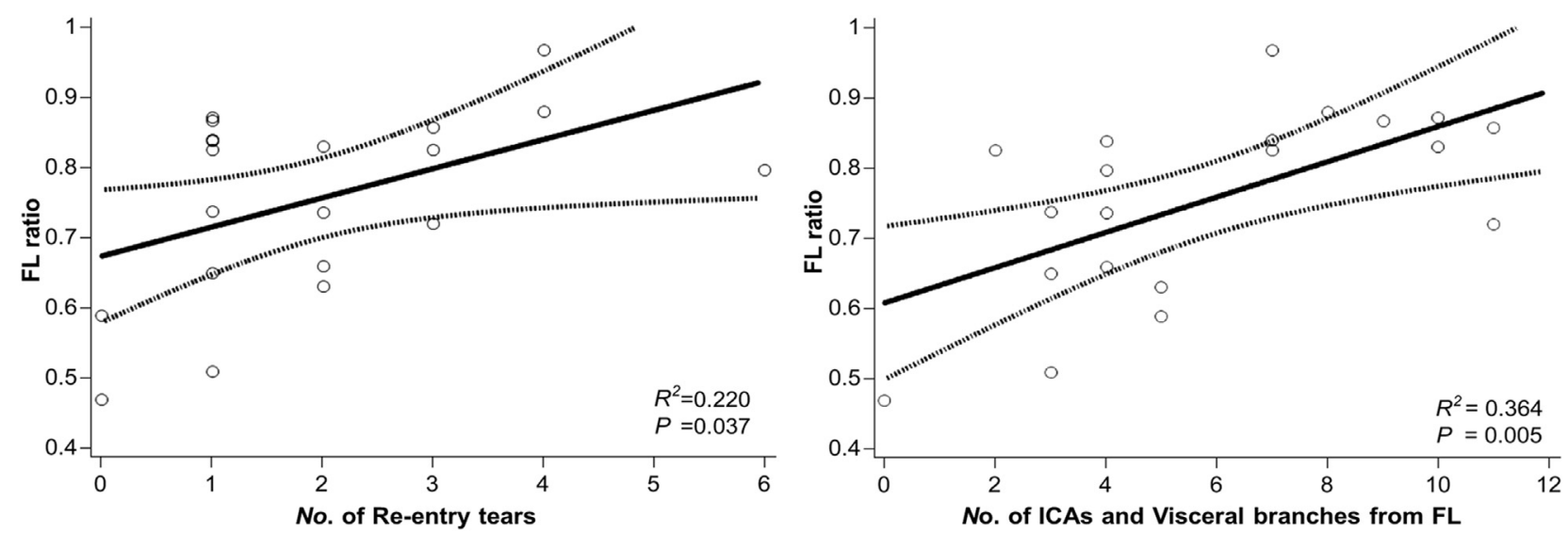

FIGURE 2. Results of univariate linear regression analysis showing prognostic factors significantly related to a decreased false lumen $(F L)$ ratio. $I C A$, Intercostal artery.

tear was sealed by the stent graft in all patients, and a mean of 3.1 tears (reentry tears, 2.0; large intimal tears, 1.1) remained below the stent graft. The number of large intimal tears found using CTA 4 weeks after TEVAR was selected as a parameter for analysis and was a significant risk factor associated with partial thrombosis in the FL $(P=.037)$.

The second mechanism proposes retrograde filling perfusion of the FL by way of the ICAs or visceral branches originating from the FL. Visceral branches solely supplied by the FL in extensive type B dissections were first emphasized by Dinsmore and colleagues. ${ }^{25}$ They proposed that if vital organs did not depend on the FL for their blood supply, complete FL occlusion tended to occur. Others ${ }^{26}$ have reported that the visceral branches that arose partially or totally from the FL were risk factors for partial thrombosis in acute DeBakey IIIb aortic dissection. To date, no report has been published about ICAs affecting aorta remodeling. The present study revealed that the number of visceral branches and ICAs arising partially or totally from the FL was an independent risk factor associated with FL shrinkage $(P=.002)$ at 4.7 month after TEVAR. The results from our study suggested the first mechanism would affect the early phase of aortic remodeling, and the second mechanism would affect aortic remodeling after the period.

Regarding the third mechanism, the role of intimal flap motion was recently analyzed in another report. ${ }^{27}$ Unimpeded septal motion could conceivably prevent flow stagnation and thrombosis in the aortic FL. The present study revealed the younger age was an important risk factor for FL shrinkage 4.7 months after TEVAR $(P=.015)$. This was because the elderly would have more restricted septal motion than would younger patients.

With our TEVAR strategy for CDIIIb aneurysms, extending the stent graft to the level of the celiac trunk, all reentry tears in the descending thoracic aorta were completely covered. This strategy resulted in complete FL thrombosis at the stent graft level in 13 patients
(65\%) during a mean follow-up duration of 28 days. Furthermore, FL thrombosis occurred more frequently in patients with fewer residual reentry tears below the level of the stent graft and in those with fewer ICAs and visceral arteries from the FL. However, the level of the distal stent graft was not associated with FL thrombosis or aorta remodeling. Although we expected the more distal landing zone would result in more complete FL thrombosis, the rate was only $37.5 \%$ in group 1 compared with $80 \%$ in group 2 in our study (Table E1). This unexpected result suggests that the level of the distal stent graft itself is not associated with the FL thrombosis. Rather, the anatomic factors such as the number of reentry tears, large intimal tears, ICAs from the FL, and visceral branches from the FL would be more important for the outcome after TEVAR in patients with CDIIIb aneurysms.

\section{Additional Concerns}

The potential for endograft collapse, stent graft-induced new intimomedial erosions, and retrograde aortic dissections are additional concerns regarding TEVAR management of CDIIIb aneurysms. ${ }^{14}$ Despite the frequent presence of small, distal, true lumens and rigid septa, we did not observe endograft collapse, perhaps because we used tapered grafts for the distal true lumens. Two patients experienced new, stent graft-induced intimal tears. Excessive oversizing of the distal stent graft could be a significant risk factor with regard to late distal intimomedial erosion. ${ }^{28}$ After those cases, we stopped using excessive oversizing and did not deploy stent grafts at the mid-descending thoracic aorta level. Similarly, retrograde dissections, reported in $\leq 4 \%$ of other cases, ${ }^{14}$ did not occur in the present series. These events underscore the limitations of the current stent graft designs for chronic dissections. In most cases, treatment extended to the left common carotid artery, and the endograft must adapt to significant differences in lumen diameters between the proximal and compressed 
distal true lumens. The next generation of stent graft design will likely have pathology-specific modifications to address these challenges. ${ }^{29}$ The most dreaded complication of TEVAR for CDIII aneurysm management is SCI. When we decide the level of distal landing zone, we should assess the balance between the benefit of complete FL thrombosis, and the risk of SCI. Although we aggressively manage CDIIIb aneurysms at our institution, deploying the stent grafts above the celiac trunk level, SCIs have not occurred. Of note, we use a protocol involving liberal use of cerebrospinal fluid drainage and subclavian revascularization.

\section{Study Limitations}

The present report had several important limitations. First, this was a single-center, retrospective analysis, without an open surgical cohort for comparison. Regardless, open therapy could still be an attractive option for selected patients. Svensson et $\mathrm{al}^{10}$ reviewed a contemporary open surgical series involving elective descending thoracic aortic aneurysm repair, including those attributed to chronic dissection, and recommended open surgical repair only in low-risk patients. Second, because of the lack of consensus regarding the definition of clinical success and favorable aortic remodeling, the present results might not be directly comparable to other reports of TEVAR for CDIII aneurysms. Mani and colleagues ${ }^{15}$ defined aorta remodeling as a $>0.5 \mathrm{~cm}$ reduction in the maximum descending thoracic aorta diameter from preoperative imaging to the final follow-up imaging study. However, we used the degree of FL thrombosis and FL ratio as surrogate markers for aorta remodeling. A consensus on more accurate classification and definition could help to determine the most appropriate method of treating chronic dissection in terms of the timing and extent of aortic coverage. ${ }^{20}$ Third, the number of patients was very small, and the follow-up duration was too short to draw conclusions regarding the aorta remodeling process and survival analysis. However, we confirmed that the period for FL thrombosis could be as short as 4 weeks and that FL regression occurred at around 3 to 6 months after TEVAR. Fourth, although postoperative CTA was available for all patients, only 2 points were analyzed to discern FL thromboses and diameters. Moreover, multilevel and volumetric data were not abstracted, which might have offered a more comprehensive and precise assessment of aortic remodeling for dissection-related pathologic features. ${ }^{16}$ FL patency status was determined using the 3 phases of postoperative CTA, and this might may not be the most sensitive or specific method for analyzing this endpoint, given the reports describing the efficacy of various dynamic magnetic resonance imaging techniques. ${ }^{27}$

\section{CONCLUSIONS}

TEVAR for CDIIIb aneurysms ensures a high degree of technical success and results in acceptable perioperative morbidity and mortality. SCI can be prevented with the liberal use of spinal drainage and subclavian revascularization. Although all reentry tears were fully covered above the celiac trunk, the visceral branches and ICAs from the FL and visible communicating channels below the celiac trunk will keep the FL pressurized and are unfavorable prognostic factors of aorta remodeling after TEVAR for CDIIIb aneurysms.

The authors deeply appreciate the participants who took part in this study and the staff of the Hybrid Aortic Center at Gangnam Severance Hospital, Yonsei University College of Medicine. We also thank Dong-Su Jang, MFA (Medical Illustrator, Medical Research Support Section, Yonsei University College of Medicine, Seoul, Korea), for the medical illustrations.

\section{References}

1. Trimarchi S, Nienaber CA, Rampoldi V, Myrmel T, Suzuki T, Bossone E, et al. Role and results of surgery in acute type B aortic dissection: insights from the International Registry of Acute Aortic Dissection (IRAD). Circulation. 2006; 114(1 Suppl):I357-64.

2. Nienaber CA, Rousseau H, Eggebrecht H, Kische S, Fattori R, Rehders TC, et al Randomized comparison of strategies for type B aortic dissection: the INvestigation of STEnt Grafts in Aortic Dissection (INSTEAD) trial. Circulation. 2009; 120:2519-28.

3. Zoli S, Etz CD, Roder F, Mueller CS, Brenner RM, Bodian CA, et al. Long-term survival after open repair of chronic distal aortic dissection. Ann Thorac Surg. 2010;89:1458-66.

4. Estrera AL, Rubenstein FS, Miller CC III, Huynh TT, Letsou GV, Safi HJ. Descending thoracic aortic aneurysm: surgical approach and treatment using the adjuncts cerebrospinal fluid drainage and distal aortic perfusion. Ann Thorac Surg. 2001;72:481-6.

5. Parsa CJ, Schroder JN, Daneshmand MA, McCann RL, Hughes GC. Midterm results for endovascular repair of complicated acute and chronic type B aortic dissection. Ann Thorac Surg. 2010;89:97-102; discussion 102-4.

6. Makaroun MS, Dillavou ED, Wheatley GH, Cambria RP; Gore TAG Investigators. Five-year results of endovascular treatment with the Gore TAG device compared with open repair of thoracic aortic aneurysms. J Vasc Surg. 2008;47: 912-8.

7. Atkins MD Jr, Black JH III, Cambria RP. Aortic dissection: perspectives in the era of stent-graft repair. J Vasc Surg. 2006;43(Suppl A):30A-43A.

8. Rodriguez JA, Olsen DM, Lucas L, Wheatley G, Ramaiah V, Diethrich EB. Aortic remodeling after endografting of thoracoabdominal aortic dissection. $J$ Vasc Surg. 2008;47:1188-94.

9. Manning BJ, Dias N, Ohrlander T, Malina M, Sonesson B, Resch T, et al. Endovascular treatment for chronic type B dissection: limitations of short stent grafts revealed at midterm follow-up. J Endovasc Ther. 2009;16:590-7.

10. Svensson LG, Kouchoukos NT, Miller DC, Bavaria JE, Coselli JS, Curi MA, et al. Expert consensus document on the treatment of descending thoracic aortic disease using endovascular stent-grafts. Ann Thorac Surg. 2008;85:S1-41.

11. Thrumurthy SG, Karthikesalingam A, Patterson BO, Holt PJ, Hinchliffe RJ Loftus IM, et al. A systematic review of mid-term outcomes of thoracic endovascular repair (TEVAR) of chronic type B aortic dissection. Eur J Vasc Endovasc Surg. 2011;42:632-47.

12. Song SW, Chang BC, Cho BK, Yi G, Youn YN, Lee S, et al. Effects of partial thrombosis on distal aorta after repair of acute DeBakey type I aortic dissection. J Thorac Cardiovasc Surg. 2010;139:841-7.

13. Mitchell RS, Ishimaru S, Ehrlich MP, Iwase T, Lauterjung L, Shimono T, et al First international summit on thoracic aortic endografting: roundtable on thoracic aortic dissection as an indication for endografting. J Endovasc Ther. 2002;9: 98-105.

14. Yang CP, Hsu CP, Chen WY, Chen IM, Weng CF, Chen CK, et al. Aortic remodeling after endovascular repair with stainless steel-based stent graft in acute and chronic type B aortic dissection. J Vasc Surg. 2012;55:1600-10.

15. Mani K, Clough RE, Lyons OT, Bell RE, Carrell TW, Zayed HA, et al. Predictors of outcome after endovascular repair for chronic type B dissection. Eur J Vasc Endovasc Surg. 2012;43:386-91. 
16. Andacheh ID, Donayre C, Othman F, Walot I, Kopchok G, White R. Patient outcomes and thoracic aortic volume and morphologic changes following thoracic endovascular aortic repair in patients with complicated chronic type B aortic dissection. J Vasc Surg. 2012;56:644-50.

17. Kim KM, Donayre CE, Reynolds TS, Kopchok GE, Walot I, Chauvapun JP, et al. Aortic remodeling, volumetric analysis, and clinical outcomes of endoluminal exclusion of acute complicated type B thoracic aortic dissections. J Vasc Surg. 2011:54:316-24.

18. Kang WC, Greenberg RK, Mastracci TM, Eagleton MJ, Hernandez AV, Pujara AC, et al. Endovascular repair of complicated chronic distal aortic dissec tions: intermediate outcomes and complications. J Thorac Cardiovasc Surg. 2011;142:1074-83

19. Virtue Registry Investigators. The VIRTUE registry of type B thoracic dissections-study design and early results. Eur J Vasc Endovasc Surg. 2011;41: 159-66.

20. Dake MD, Thompson M, van Sambeek M, Vermassen F, Morales JP. DISSECT: a new mnemonic-based approach to the categorization of aortic dissection. Eur J Vasc Endovasc Surg. 2013;46:175-90.

21. Manning BJ, Dias N, Manno M, Ohrlander T, Malina M, Sonesson B, et al. Endovascular treatment of acute complicated type B dissection: morphological changes at midterm follow-up. J Endovasc Ther. 2009;16:466-74.

22. Qing KX, Yiu WK, Cheng SW. A morphologic study of chronic type B aortic dissections and aneurysms after thoracic endovascular stent grafting. $J$ Vasc Surg. 2012;55:1268-75.

23. Quint LE, Platt JF, Sonnad SS, Deeb GM, Williams DM. Aortic intimal tears: detection with spiral computed tomography. J Endovasc Ther. 2003;10:505-10.

24. Khoynezhad A, Walot I, Kruse MJ, Rapae T, Donayre CE, White RA. Distribution of intimomedial tears in patients with type B aortic dissection. $J$ Vasc Surg. 2010;52:562-8.

25. Dinsmore RE, Willerson JT, Buckley MJ. Dissecting aneurysm of the aorta: aortographic features affecting prognosis. Radiology. 1972;105:567-72.

26. Qin YL, Deng G, Li TX, Jing RW, Teng GJ. Risk factors of incomplete thrombosis in the false lumen after endovascular treatment of extensive acute type B aortic dissection. J Vasc Surg. 2012;56:1232-8.

27. Karmonik C, Duran C, Shah DJ, Anaya-Ayala JE, Davies MG, Lumsden AB, et al. Preliminary findings in quantification of changes in septal motion during follow-up of type B aortic dissections. J Vasc Surg. 2012;55:1419-26.

28. Huang CY, Weng SH, Weng CF, Chen WY, Chen IM, Hsu CP, et al. Factors predictive of distal stent graft-induced new entry after hybrid arch elephant trunk repair with stainless steel-based device in aortic dissection. J Thorac Cardiovasc Surg. 2013;146:623-30

29. Nienaber CA, Kische S, Zeller T, Rehders TC, Schneider H, Lorenzen B, et al. Provisional extension to induce complete attachment after stent graft placement in type B aortic dissection: the PETTICOAT concept. J Endovasc Ther. 2006;13: 738-46.

\section{Discussion}

Dr John S. Ikonomidis (Charleston, SC). Congratulations on a very interesting study that is advancing our knowledge about the predictive factors for remodeling and FL thrombosis. I wonder if you could reconcile a few issues for me.

You defined partial thrombosis as a situation in which both flow and thrombus are present in the FL, but I wonder whether this is a specific enough definition. I wonder if you would comment on exactly what you mean by flow. For example, from the CT scans that you have, if you see a patient with a simple type I endoleak, are you defining that as flow?

Dr Song. Yes. Thank you for your comments, Dr Ikonomidis.

Regarding the FL status, if some contrast is present in the FL with the arterial phase or delayed phase, we regard the status of the FL as partial thrombosis. If we cannot find any contrast in the arterial phase and delayed phase, we regard it as complete thrombosis.

In other words, we regard the contrast as flow. But the CTA might not be the most sensitive or specific method for analyzing this endpoint given the reports about various dynamic magnetic resonance imaging techniques.

Also, in our study, no type Ia endoleaks occurred. Thus, the FL will be thrombosed from top to bottom. I think that immediately after TEVAR, antegrade flow or retrograde flow is always present to the FL in the distal part. If it has not disappeared at the latest surveillance scan, it can be classified as partial thrombosis.

Dr Ikonomidis. Thank you. Second, in the Gangnam TEVAR registry, you identified 20 of 103 patients who had undergone elective TEVAR. How many patients in that registry had chronic type $\mathrm{B}$ dissections that were not included in this analysis from that registry? Also, did they undergo stent grafting, or, if not, what was the reason that they were not included?

Dr Song. Yes. The Gangnam TEVAR registry identified 103 patients who had undergone elective TEVAR within a 1-year period. Among those, about 40 patients had a degenerative aortic aneurysm, 30 had acute type $\mathrm{B}$ aortic dissection, and 10 had traumatic dissections.

Also, we are following up many patients with chronic type B dissection by ourselves, and if the aneurysm expands $>5.5 \mathrm{~cm}$ or the growth rate is $>5 \mathrm{~mm}$ in 6 months or if newly developed back pain or continuous pain or malperfusion is present, that is an indication for elective TEVAR.

Also, during this study period, 4 patients underwent surgical repair of the thoracoabdominal aorta. All these patients were very young or had some connective tissue disease or concomitant enlargement of a dissecting aneurysm at the abdominal aorta.

Dr Ikonomidis. Thank you. My last question pertains to the report, in which you state that in all patients, full coverage of the descending thoracic aorta was achieved. However, in this particular study, it becomes apparent that there was progression in the distal landing zone, first in the mid-descending thoracic aorta and progressing distally such that in some patients juxtaceliac coverage was present.

Could you comment on the differences in the distal landing zone and did that happen as a result of the anatomy that you saw or as the result of a progressive change in your selection of the distal landing zone?

Dr Song. Yes. I apologize that I have made some confusion for you, but our basic principle for TEVAR for the patient with a complicated type IIIb aortic aneurysm is to cover all the visible communicating channels at the descending thoracic aorta.

For the 2 patients in group 3 whose distal landing zone was at the mid-descending thoracic aorta, no visible tears were seen on a CTA at the distal part of the descending thoracic aorta.

We experienced new intimal tears at the end of the stent graft in the 2 patients. After that case, we decided not to deploy any more stents at the mid-descending thoracic aorta level but to cover the whole descending thoracic aorta and treat down to just above the celiac trunk. It was our intent to cover all the visible and invisible communicating channels, if possible.

Dr Ikonomidis. Thank you very much, and, once again, congratulations for a nicely performed and presented study.

Dr Song. Thank you.

Dr Hisham M. F. Sherif (Newark, Del). I have no financial disclosures. Thanks very much for your nice presentation.

You state that one of the reasons for aortic remodeling is persistence of intercostal segmental artery flow, which technically constitutes a type II endoleak. 
My 2 questions are, first, at the time of deployment of the endograft, was that recognized, and if so, was it addressed at the time? Also, if it has not been addressed, are you planning to follow-up on this endoleak with another study to see whether other measures have eliminated it or not?

Dr Song. Thank you for your excellent questions. However, very few reports have been published about type II endoleaks for chronic type III aortic dissections.

In a case with acute type B dissections, 1 study commented on the visceral branches for the backflow from the visceral branch to the FL. However, as far as I know, no report has yet been published about ICAs from the FL that make retrograde backflow to fill the FL and prevent FL thrombosis. I think it is very difficult to know if a type II endoleak from the ICAs is present at intervention. Also, I cannot tell you about the type II endoleak, which is a terminology frequently used with the degenerative aneurysm, because the pathologic features are clearly different between chronic IIIb aneurysm and degenerative aneurysm.

Dr Sherif. Thanks for the presentation. 

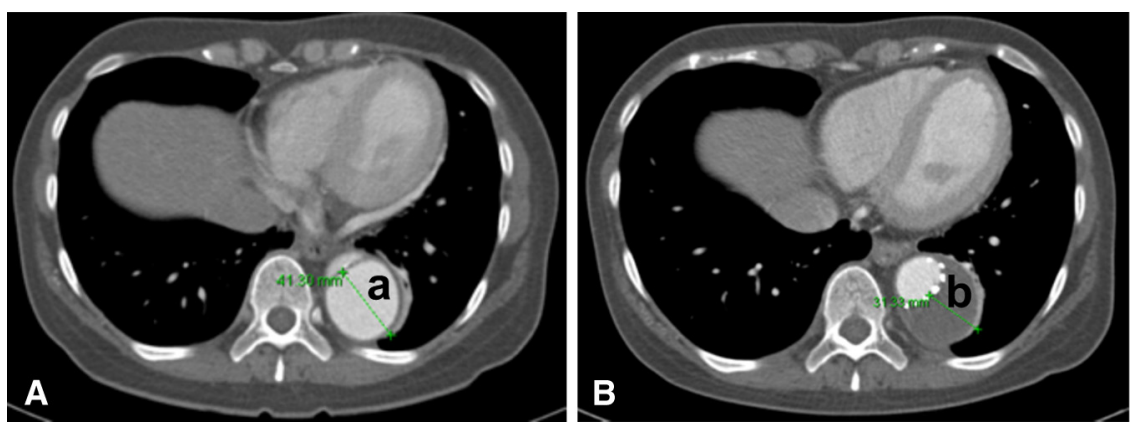

FIGURE E1. Aortic remodeling analysis using preoperative and postoperative computed tomography angiograms (CTAs). A, Axial view of the preoperative CTA. B, Axial view of the postoperative CTA. $a$, Maximal diameter of the false lumen on the preoperative CTA; $b$, maximal diameter of the false lumen on the postoperative CTA.
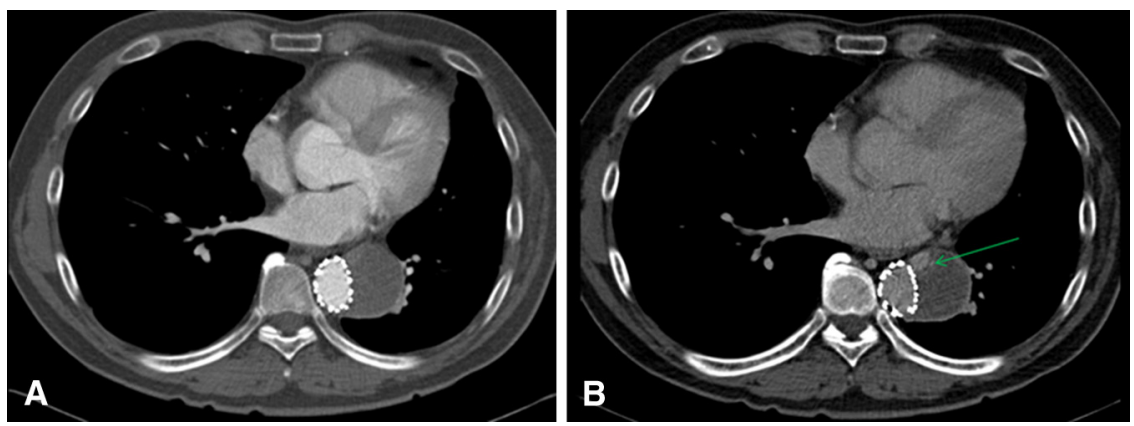

FIGURE E2. Alteration of false lumen thrombosis according to the phase of the postoperative computed tomography angiogram (CTA). A, Axial view of the CTA showing complete thrombosis in the arterial phase. B, Axial view of the CTA, at the same level, showing partial thrombosis in the delayed phase.

TABLE E1. Distal landing zone

\begin{tabular}{|c|c|c|c|c|}
\hline Variable & Group $1(n=8)$ & Group $2(n=10)$ & Group $3(n=2)$ & $P$ value \\
\hline Spinal drainage & $8(100)$ & $5(50)$ & $0(0)$ & .006 \\
\hline Reentry & $2.1 \pm 1.4$ & $1.9 \pm 1.8$ & $1.5 \pm 0.7$ & .874 \\
\hline Large intimal tear & $1.4 \pm 0.9$ & $0.9 \pm 0.6$ & $1.0 \pm 1.4$ & .462 \\
\hline ICAs from FL & $5.1 \pm 3.4$ & $3.4 \pm 2.6$ & $5.0 \pm 4.2$ & .477 \\
\hline Visceral branches from FL & $1.6 \pm 1.1$ & $1.6 \pm 1.1$ & $1.5 \pm 0.7$ & .989 \\
\hline FL ratio & $0.74 \pm 0.14$ & $0.76 \pm 0.14$ & $0.76 \pm 0.13$ & .902 \\
\hline Complete thrombosis & $3(37.5)$ & $8(80)$ & $2(100)$ & .147 \\
\hline
\end{tabular}

Data presented as $\mathrm{n}(\%)$ or mean \pm standard deviation. ICA, Intercostal artery; $F L$, false lumen. 